\title{
Sarcomas - the model for multidisciplinary care in cancer
}

\author{
Ian Judson \\ Emeritus Professor of Cancer Pharmacology, The Institute of Cancer Research, Sutton, Surrey SM2 5NG, UK.
}

Correspondence to: Prof. Ian Judson, E-mail: lan.Judson@icr.ac.uk

How to cite this article: Judson I. Sarcomas - the model for multidisciplinary care in cancer. J Cancer Metastasis Treat 2021;7:28. https://dx.doi.org/10.20517/2394-4722.2021.110

Received: 6 May 2021 Accepted: 12 May 2021 Published: 25 May 2021

Academic Editor: Lucio Miele Copy Editor: Yue-Yue Zhang Production Editor: Yue-Yue Zhang

Sarcomas were one of the first cancers for which multidisciplinary clinics and team meetings were set up, acknowledging the need for all the diagnostic and treatment disciplines to be involved in the clinical decision-making process. This is amply demonstrated by the papers chosen for this special issue of the Journal of Cancer Metastasis and Treatment, which includes articles on basic biology, translational research, pathology, surgery and survivorship.

Sarcomas represent a bewildering collection of more than a hundred different clinical entities, each having its own characteristics in terms of prognosis, preferred site of origin, pattern of metastatic spread, responsiveness to therapy and molecular drivers. Molecularly targeted therapy of sarcomas began, of course, with the use of imatinib in the treatment of gastrointestinal stromal tumour ${ }^{[1]}$, where the driver is usually a mutant form of KIT, which is permanently switched on. Unfortunately, this proved to be somewhat of an outlier, and although imatinib has been found to be useful in a limited number of other sarcomas driven by gene fusions, this drug is not of general utility. However, as happened in the field of haematology some years ago, sarcomas are now increasingly being defined by their molecular characteristics, such as driver mutations or chromosomal translocations. Although the $\mathrm{t}(\mathrm{X} ; 18)$ translocation that characterises synovial sarcoma was first described over 33 years ago ${ }^{[2]}$, and the genes involved, SYT and SSX1/2, were identified some years later ${ }^{[3]}$, it is only quite recently that the molecular mechanisms by which the translocation alters the transcription of multiple genes have begun to be revealed ${ }^{[4,5]}$. Although a number of promising lines of investigation are now being pursued, this information has not yet led to treatment specifically targeting the 
translocation.

The latest edition of the WHO Classification of Tumors of Soft Tissue and Bone, published in 2020, lists many more sarcomas that are now classified by their molecular characteristics, such as those driven by a CIC-DUX4 fusion gene, previously a subset of Ewing sarcoma-like small round cell tumours lacking an EWSR1 fusion. While it is now known that this is a distinct entity, characterised by an aggressive clinical course and poor response to chemotherapy ${ }^{[6]}$, this knowledge has not resulted in a targeted therapy. The synovial sarcoma story warns us to be patient.

There are some recent successes, for example the use of ALK inhibitors such as crizotinib in the treatment of inflammatory myofibroblastic tumour ${ }^{[7]}$, and the approval by the FDA of the EZH2 inhibitor tazemetostat for the treatment of epithelioid sarcoma following the report of responses and improved survival in a basket trial ${ }^{[8]}$. In this current issue devoted to the topic of sarcomas we have a number of articles also relating to the identification of known or potential new targets for therapy, but also papers on surgical technique and survivorship.

The paper by Karolak and colleagues ${ }^{[9]}$ focuses on the role of EZH2, a histone methyltransferase which is a component of the chromatin-modifying complex PRC2 (Polycomb Repressive Complex 2). EZH2 is responsible for adding methyl groups to Lysine 27 on histone $\mathrm{H} 3$. The resultant $\mathrm{H} 3 \mathrm{~K} 27 \mathrm{Me} 3$ mark is an important repressive signal, resulting in condensation of chromatin and inhibiting transcription of the associated gene(s). A number of sarcomas, such as MPNST, synovial sarcoma, leiomyosarcoma, epithelioid sarcoma and rhabdomyosarcoma are associated with overexpression of $\mathrm{EZH} 2$.

Overexpression is associated with a worse prognosis and a higher rate of distant metastasis. The effects of EZH2 activity include inhibition of terminal differentiation and senescence and maintenance of proliferation. Some sarcomas are characterised by loss of a component of the SW1/SNF chromatinremodelling complex, SMARCB1, also known as INI1. SW1/SNF has opposing activity to PRC2 and mutations in SMARCB1 may result in loss of function of this subunit, hence of the SW1/SNF complex resulting in effective upregulation of $\mathrm{PRC} 2$, hence $\mathrm{EZH} 2$. The $\mathrm{EZH} 2$ inhibitor tazemetostat has demonstrated significant activity against epithelioid sarcoma, a diseases for which loss of INI1 has long been known to be a diagnostic marker, with a $15 \%$ response rate and median overall survival of 19 months ${ }^{[8]}$. In the same study patients with synovial sarcoma, also with INI1 loss, were included but best response was stable disease (SD), with $15 \%$ of patients having durable SD for $>16$ weeks. Loss of INI1 is clearly insufficient to predict substantial anti-tumour activity with $\mathrm{EZH} 2$ inhibition. Laboratory studies appear to indicate that downregulation of $\mathrm{EZH} 2$ inhibits tumour growth in tumours with $\mathrm{EZH} 2$ overexpression, but this may not consistently translate into activity with EZH2 inhibitors in the clinic.

The paper by Abe et al. ${ }^{[10]}$ reviews the role of glycogen synthase kinase $3 \beta$ (GSK3 $\beta$ ) in sarcomas. GSK3 $\beta$ is an enzyme that phosphorylates glycogen synthase, and other important proteins, on serine and threonine residues. There is growing evidence that GSK3 $\beta$ can play a role in tumorigenesis and extensive investigations have confirmed it as a potential therapeutic target in a number of different malignancies ${ }^{[11]}$. One of the key mechanisms appears to be positive transcriptional control of NF- $\mathrm{kB}$ and subsequent cancer cell survival and resistance to chemotherapy. In their review of the data linking GSK3 $\beta$ expression and activation to sarcomas Abe et al. ${ }^{[10]}$ first discuss the knowledge to date on molecularly targeted therapy of sarcomas and makes a persuasive case that GSK3 $\beta$ plays an important role in a number of sarcomas and may be a useful target for novel therapy. In osteosarcoma, a disease sorely in need of new treatment, the active form of GSK3 $\beta$ is overexpressed compared to normal osteoblasts and inhibition results in reduced 
tumour cell survival and increased expression of $\beta$-catenin, a potential tumour suppressor in this disease. Inhibition of GSK3 $\beta$ can induce apoptosis in osteosarcoma cells.

Other sarcomas in which GSK3 $\beta$ has been shown to play a potential role include rhabdomyosarcoma, again possibly via the $\beta$-catenin pathway, synovial sarcoma, fibrosarcoma and undifferentiated pleomorphic sarcoma. GSK3 $\beta$ may also be involved in tumour-induced suppression of the immune response as well as being involved in doxorubicin-mediated cardiotoxicity, chemotherapy-associated neuropathy and impaired tissue repair. Inhibitors of GSK3 $\beta$ have been demonstrated to have antitumour effects in vivo in osteosarcoma ${ }^{[12]}$ synovial sarcoma and fibrosarcoma xenografts ${ }^{[1.3]}$. To date there have been no clinical trials of GSK3 $\beta$ inhibitors in sarcoma but these data would appear to justify such a study.

Another potential molecular target for sarcomas that involves the WNT pathway, but in this case the noncanonical $\beta$-catenin independent pathway, is ROR2 (receptor tyrosine kinase-like orphan receptor 2 ). In the paper by Tran et al. ${ }^{[14]}$ in this issue, they describe the ability of ROR2 to inhibit anoikis, and hence increase the rate of pulmonary metastases in a mouse model of metastatic osteosarcoma, a process that involves activation of AKT. It is suggested that AKT inhibitors currently in clinical development might play a role in combatting the development of lung metastases in this disease. It is not clear whether this strategy would have any utility against established micrometastases. Clinical trials investigating treatment to prevent the development of metastases are hard to design. However, this pathway is worthy of further study and may shed further light on the metastatic process in osteosarcoma.

The next two papers concern different aspects of orthopaedic oncology. The first, by Pacheco and Righi ${ }^{[15]}$ from the Rizzoli Institute in Bologna, reviews malignant tumours of the bone surface. All sarcoma clinicians will be familiar with the distinction between parosteal osteosarcoma and conventional high grade osteosarcoma in terms of prognosis and need for intensive chemotherapy, in that the rare parosteal variant has a good prognosis and does not require treatment with chemotherapy unless it differentiates, in which case the prognosis is much worse. It is interesting to note that parosteal osteosarcoma is associated with chromosomal amplification involving both the MDM2 and CDK4 genes, something it shares with welldifferentiated or dedifferentiated liposarcoma. They may be less familiar with periosteal osteosarcoma and bone surface tumours of cartilaginous origins, such as secondary peripheral chondrosarcoma, which originates in osteochondromas, and periosteal chondrosarcoma. All of these rare entities are very well described, in terms of differential diagnosis, along with appropriate radiological and histological illustrations, prognosis and management. The need for specialised multidisciplinary care is emphasised, as is appropriate.

The paper by Pinnameni and Damron ${ }^{[16]}$ concerns reconstructive procedures for the proximal humerus in the cancer setting. Techniques for reconstruction in the pelvis, including hip replacement, a common procedure outside orthopaedic oncology, are well established. The proximal humerus represents a much more difficult technical challenge not least because of the proximity of major nerves and blood vessels but also the fact that stability of the glenohumeral joint is dependent on the surrounding muscles, which may need to be sacrificed in a cancer operation. This paper deals with the indications for surgery, both primary and secondary, the various techniques available, together with their pros and cons, both short term and long term and recent developments in technology, including prosthetics. The paper concludes with the fact that comparative studies are lacking to define the most appropriate reconstructive procedures, something that only prospective clinical trials could achieve. 
The study by Baker et al. ${ }^{[17]}$ concerns survivorship. Doxorubicin remains a staple component of chemotherapy for metastatic sarcoma and those tumours for which intensive multi-agent chemotherapy is given with curative intent, such as Ewing sarcoma and osteosarcoma. A recent study in the pre-operative setting indicated that for a number of different high grade extremity soft tissue sarcoma histotypes, supposedly histotype-tailored chemotherapy regimens proved inferior to a combination of ifosfamide and an anthracycline (epirubicin) in terms of disease-free and overall survival, although the paper was unable to draw definitive conclusions owing to its statistical design ${ }^{[18]}$. The reason for the superior efficacy of anthracycline/doxorubicin in the treatment of sarcomas remains unclear.

Given that treatment with doxorubicin remains necessary, it is extremely valuable to examine the long term consequences of anthracycline use in survivors and to study ways in which the adverse effects can be mitigated. Historically the focus has been on the cardiomyopathy associated with doxorubicin, the risk of which relates to the cumulative dose and can be ameliorated by cumulative dose restriction and the use of dexrazoxane, as confirmed by randomised clinical trials ${ }^{[19]}$ and a number of studies in different diseases, including osteosarcoma ${ }^{[20]}$. These studies not only demonstrated significant cardioprotection but no reduction in disease control nor an increased incidence of secondary malignancy. Note in their paper on GSK $3 \beta$ Abe et al. ${ }^{[10]}$ fail to mention dexrazoxane in their discussion of the potential for GSK $3 \beta$ inhibitors to exert a cardioprotective effect, which is an important omission.

However, cardiomyopathy is not the only problem. Cancer survivors treated with anthracyclines have a much increased risk of coronary artery disease (CAD) and also of a number of the chronic diseases which are known risk factors for $\mathrm{CAD}$, such as hypertension, obesity, diabetes and dyslipidaemia. In a prospective 5 -year study, Baker et al. ${ }^{[17]}$ followed 61 cancer survivors with a median age of 42 years who had received adjuvant or neoadjuvant doxorubicin. Data on known risk factors were collected at baseline and prospectively, including high body mass index, smoking and alcohol use, exercise, hypertension, elevated cholesterol, increased high-sensitivity C-reactive protein, an index of inflammation and known CAD risk factor, type 2 diabetes, depression and anxiety, and impaired renal function. The findings indicate a much higher incidence of risk factors for CAD than in the general population, even in patients $<40$ years of age, and the need for active intervention to mitigate known risk factors where possible. The authors strongly recommend that cancer survivors be followed in oncology clinics. This may not always be feasible and will vary in different healthcare jurisdictions, but perhaps shared care arrangements with primary care together with clear guidance from the cancer service as to what to monitor and the types of intervention required could be developed. As more and more patients survive cancer this need is bound to increase and there is an obligation to treat not just the cancer itself but also the chronic effects of therapy.

Sarcoma care remains under-resourced and under-researched and it is gratifying to see it highlighted by this interesting collection of papers.

\section{DECLARATIONS}

\section{Authors' contribution}

Judson I contributed solely to the article.

\section{Availability of data and materials}

Not applicable.

\section{Financial support and sponsorship}

None. 


\section{Conflicts of interest}

The author declares that there are no conflicts of interest.

\section{Ethical approval and consent to participate \\ Not applicable.}

\section{Consent for publication}

Not applicable.

\section{Copyright}

(c) The Author(s) 2021.

\section{REFERENCES}

1. Demetri GD, von Mehren M, Blanke CD, et al. Efficacy and safety of imatinib mesylate in advanced gastrointestinal stromal tumors. $N$ Engl J Med 2002;347:472-80. DOI PubMed

2. Smith S, Reeves BR, Wong L, Fisher C. A consistent chromosome translocation in synovial sarcoma. Cancer Genet Cytogenet 1987;26:179-80. DOI PubMed

3. Crew AJ, Clark J, Fisher C, et al. Fusion of SYT to two genes, SSX1 and SSX2, encoding proteins with homology to the Kruppelassociated box in human synovial sarcoma. EMBOJ 1995;14:2333-40. PubMed PMC

4. Kadoch C, Crabtree GR. Reversible disruption of mSWI/SNF (BAF) complexes by the SS18-SSX oncogenic fusion in synovial sarcoma. Cell 2013;153:71-85. DOI PubMed PMC

5. McBride MJ, Pulice JL, Beird HC, et al. The SS18-SSX fusion oncoprotein hijacks BAF complex targeting and function to drive synovial sarcoma. Cancer Cell 2018;33:1128-41.e7. DOI PubMed PMC

6. Antonescu CR, Owosho AA, Zhang L, et al. Sarcomas With CIC-rearrangements are a distinct pathologic entity with aggressive outcome: a clinicopathologic and molecular study of 115 cases. Am J Surg Pathol 2017;41:941-9. DOI PubMed PMC

7. Schöffski P, Sufliarsky J, Gelderblom H, et al. Crizotinib in patients with advanced, inoperable inflammatory myofibroblastic tumours with and without anaplastic lymphoma kinase gene alterations (European Organisation for Research and Treatment of Cancer 90101 CREATE): a multicentre, single-drug, prospective, non-randomised phase 2 trial. Lancet Respir Med 2018;6:431-41. DOI PubMed

8. Gounder M, Schöffski P, Jones RL, et al. Tazemetostat in advanced epithelioid sarcoma with loss of INI1/SMARCB1: an international, open-label, phase 2 basket study. Lancet Oncol 2020;21:1423-32. DOI PubMed

9. Karolak M, Tracey I, Shipley J, Walters ZS. Targeting EZH2 for the treatment of soft tissue sarcomas. J Cancer Metastasis Treat 2021;7:15. DOI

10. Abe K, Shimozaki S, Domoto T, et al. Glycogen synthase kinase $3 ß$ biology in bone and soft tissue sarcomas. J Cancer Metastasis Treat 2020;6:51. DOI

11. Walz A, Ugolkov A, Chandra S, et al. Molecular pathways: revisiting glycogen synthase kinase-3beta as a target for the treatment of cancer. Clin Cancer Res 2017;23:1891-7. DOI PubMed PMC

12. Shimozaki S, Yamamoto N, Domoto T, et al. Efficacy of glycogen synthase kinase-3beta targeting against osteosarcoma via activation of beta-catenin. Oncotarget 2016;7:77038-51. DOI PubMed PMC

13. Abe K, Yamamoto N, Domoto T, et al. Glycogen synthase kinase 3beta as a potential therapeutic target in synovial sarcoma and fibrosarcoma. Cancer Sci 2020;111:429-40. DOI PubMed PMC

14. Tran DTP, Kuchimaru T, Pongsuchart M, et al. ROR2 regulates the survival of murine osteosarcoma cells in lung capillaries. $J$ Cancer Metastasis Treat 2020;6:47. DOI

15. Pacheco M, Righi A. Primary malignant tumors of bone surface: a review with emphasis on differential diagnosis. J Cancer Metastasis Treat 2020;6:49. DOI

16. Pinanamaneni S, Damron TA. Proximal humerus reconstruction in orthopaedic oncology. J Cancer Metastasis Treat 2021;7:7. DOI

17. Baker LH, Boonstra PS, Reinke DK. Burden of chronic diseases among sarcoma survivors treated with anthracycline chemotherapy: results from an observational study. J Cancer Metastasis Treat 2020;6:24. DOI

18. Gronchi A, Ferrari S, Quagliuolo V, et al. Histotype-tailored neoadjuvant chemotherapy versus standard chemotherapy in patients with high-risk soft-tissue sarcomas (ISG-STS 1001): an international, open-label, randomised, controlled, phase 3, multicentre trial. Lancet Oncol 2017;18:812-22. DOI PubMed

19. Lipshultz SE, Scully RE, Lipsitz SR, et al. Assessment of dexrazoxane as a cardioprotectant in doxorubicin-treated children with highrisk acute lymphoblastic leukaemia: long-term follow-up of a prospective, randomised, multicentre trial. Lancet Oncol 2010;11:95061. DOI PubMed PMC

20. Kopp LM, Womer RB, Schwartz CL, et al. Effects of dexrazoxane on doxorubicin-related cardiotoxicity and second malignant neoplasms in children with osteosarcoma: a report from the Children's Oncology Group. Cardiooncology 2019;5:15. DOI PubMed PMC 